\title{
Safety of surgical tracheostomy under continued antithrombotic therapy
}

\author{
Takayuki Sugaya ${ }^{1}$, Rumi Ueha ${ }^{1}$, Taku Sato ${ }^{1}$, Takao Goto ${ }^{1}$, Akihito Yamauchi ${ }^{1}$, and \\ Tatsuya Yamasoba ${ }^{1}$ \\ ${ }^{1}$ The University of Tokyo
}

July 17, 2021

\begin{abstract}
Abstract Objective: Although various guidelines have been established for the management of antithrombotic therapy during surgical treatments, surgical tracheostomy (ST) under continued antithrombotic therapy (CAT) remains a challenge. We investigated the risk factors for complications after ST by focusing on CAT use during ST. Method: Patients' medical records from 2009 to 2020 were reviewed in this retrospective study. We selected patients who underwent ST at the Department of Otolaryngology of the University of Tokyo Hospital. Patient demographics, complications, and blood test values were recorded and statistically analyzed to identify the risk factors for postoperative complications. Results: We identified 288 patients (median age: 64 years; 184 men [64\%]), among whom 40 (median age: 67 years; 29 men [73\%]) underwent CAT. Although the patients undergoing CAT had significantly higher values of activated partial thromboplastin time $(\mathrm{p}=0.002)$ and prothrombin time-international normalized ratio $(\mathrm{p}=0.006)$ than those of antithrombotic naïve patients, no statistically significant intergroup differences were observed in the risks of bleeding, infection, or subcutaneous emphysema. Instead, ST under local anesthesia $(\mathrm{p}=0.01)$ and ST for airway emergency $(\mathrm{p}=0.02)$ significantly increased the risk of early postoperative complications. Conclusion: The results of the present study suggest that ST under CAT can be safely performed without any increased risk of postoperative complications. Nevertheless, surgeons should be extra cautious about early complications after ST under local anesthesia without intubation or ST for airway emergency. Key points: 1. We aimed to investigate the risk factors for complications after ST by focusing on CAT use during ST. 2. Patients undergoing CAT had significantly higher values of APTT and PT-INR than those of antithrombotic naïve patients. 3. ST under CAT can be safely performed without any increased risk of postoperative complications. 4. ST under local anesthesia and airway emergency was a risk factor for complications after ST. 5. Among the complications, subcutaneous emphysema was significantly more frequent in ST under local anesthesia and under airway emergency.
\end{abstract}

\section{Abstract}

Objective : Although various guidelines have been established for the management of antithrombotic therapy during surgical treatments, surgical tracheostomy (ST) under continued antithrombotic therapy (CAT) remains a challenge. We investigated the risk factors for complications after ST by focusing on CAT use during ST.

Method : Patients' medical records from 2009 to 2020 were reviewed in this retrospective study. We selected patients who underwent ST at the Department of Otolaryngology of our hospital. Patient demographics, complications, and blood test values were recorded and statistically analyzed to identify the risk factors for postoperative complications.

Results : We identified 288 patients (median age: 64 years; 184 men [64\%]), among whom 40 (median age: 67 years; 29 men [73\%]) underwent CAT. Although the patients undergoing CAT had significantly higher values of activated partial thromboplastin time $(\mathrm{p}=0.002)$ and prothrombin time-international normalized ratio $(\mathrm{p}$ 
$=0.006)$ than those of antithrombotic naïve patients, no statistically significant intergroup differences were observed in the risks of bleeding, infection, or subcutaneous emphysema. Instead, ST under local anesthesia $(\mathrm{p}=0.01)$ and ST for airway emergency $(\mathrm{p}=0.02)$ significantly increased the risk of early postoperative complications.

Conclusion : The results of the present study suggest that ST under CAT can be safely performed without any increased risk of postoperative complications. Nevertheless, surgeons should be extra cautious about early complications after ST under local anesthesia without intubation or ST for airway emergency.

\section{Key points :}

1. We aimed to investigate the risk factors for complications after ST by focusing on CAT use during ST.

2. Patients undergoing CAT had significantly higher values of APTT and PT-INR than those of antithrombotic naïve patients.

3. ST under CAT can be safely performed without any increased risk of postoperative complications.

4. ST under local anesthesia and airway emergency was a risk factor for complications after ST.

5. Among the complications, subcutaneous emphysema was significantly more frequent in ST under local anesthesia and under airway emergency.

\section{INTRODUCTION}

Surgical tracheostomy (ST) is one of the most frequently performed surgical procedures for patients with severe or critical conditions. ${ }^{1}$ Similar to any other invasive procedure, ST can result in numerous potential complications. These include early complications, such as bleeding, subcutaneous emphysema, stomal infection, and accidental decannulation, ${ }^{2}$ and late complications, such as airway stenosis, tracheomalacia, tracheocutaneous fistula, and pneumonia. ${ }^{1,2}$ Postoperative complications of ST are rare, and the early and late postoperative complication rates are $5.6 \%$ and $7.1 \%$, respectively. ${ }^{3}$ Obesity and awake tracheostomy are major risk factors for postoperative complications of ST. ${ }^{4,5}$ Further, four or more comorbidities are reported to increase the risk of postoperative bleeding, which is the most frequent early complication of ST. ${ }^{3,6}$ Additionally, ST is sometimes necessary for patients undergoing continued antithrombotic therapy (CAT), which can exacerbate the risk of bleeding. However, there is a dearth of data regarding ST under CAT.

Several guidelines recommend that tooth extraction in patients on antithrombotic therapy can be performed without reducing or discontinuing antiplatelet or anticoagulant drugs ${ }^{7-10}$ and that percutaneous endoscopic gastrostomy ${ }^{11}$ and endoscopic variceal ligation ${ }^{12}$ can be performed without discontinuing aspirin. However, no established guidelines are currently available for the management of antithrombotic therapy in patients undergoing ST. In this study, we investigated the risk factors for complications after ST by focusing on CAT use during ST.

\section{MATERIALS AND METHODS}

\subsection{Patients and ethics approval}

We included patients who underwent ST by otolaryngologists at our hospital between 2009 and 2020. Patients who underwent percutaneous tracheostomy were excluded. This study was approved by the Human Ethics Committee of our hospital. Written informed consent was obtained from every patient, and patient anonymity was preserved.

\subsection{Methodology}

We conducted a retrospective, single-center study using medical charts from the hospital database of our institute. The clinical and demographic profiles of the patients, including age, sex, and body mass index; 
presence of diabetes mellitus (DM), hypertension, primary diseases, or airway emergency; method of anesthesia (local anesthesia without intubation or general anesthesia); use of antithrombotic drugs; presence of complications; blood test values, were analyzed.

Complications were reviewed with respect to the incidences of bleeding, infection, and subcutaneous emphysema. We defined "bleeding" as bleeding lasting 1 week after ST or bleeding that required surgical hemostasis; "infection" as a condition requiring the application of antibiotic ointments or surgical treatment, including the excision of the infected area; "subcutaneous emphysema" as the evident presence of subcutaneous emphysema around the tracheostoma.

Blood test values were reviewed to determine the albumin level, lymphocyte count, platelet count, prothrombin time (PT), activated partial thromboplastin time (APTT), and prothrombin time-international normalized ratio (PT-INR). The abnormal ranges for albumin level, lymphocyte count, platelet count, PT, APTT, and PT-INR were defined as $<3.0 \mathrm{~g} / \mathrm{dL},<1500 / \mu \mathrm{L},<50000 / \mu \mathrm{L},>14 \mathrm{~s},>40 \mathrm{~s}$, and $>1.5$, respectively.

We also extracted the data of patients who underwent ST under CAT. Thereafter, we compared the patient backgrounds and risks of complications after ST between the antithrombotic naïve patients and patients undergoing CAT. Furthermore, we analyzed the risk factors for complications after ST.

\subsection{Statistical analyses}

We analyzed the data using BellCurve for Excel (version 3.20, Social Survey Research Information Co., Ltd., Tokyo, Japan). To compare the antithrombotic naïve patients and patients undergoing CAT to patients with or without postoperative complications, we used the Mann-Whitney U test for continuous variables and Fisher's exact test or chi-square test for categorical variables. A p value $<0.05$ was considered statistically significant.

\section{RESULTS}

\subsection{Patient demographics}

Table 1 lists the demographic data of the enrolled patients. We identified 288 eligible patients. Their median age at the time of tracheostomy was 64 years (interquartile range, 48-73 years), and there was a male predominance $(64 \%)$. The most common primary diseases were cerebrovascular and neuromuscular diseases $(26 \%)$. The primary reasons for tracheostomy were long-term intubation (72\%), upper-airway narrowing (21\%), and difficult intubation (6\%). Among them, 39 patients (14\%) underwent emergency ST. ST was performed under local anesthesia in 57 patients (19\%). ST under CAT was performed in 40 patients (14\%).

\subsection{Effects of antithrombotic use on complications after ST}

The background of patients according to their CAT status is summarized in Table 2. Patients undergoing CAT had significantly higher values of APTT $(\mathrm{p}=0.002)$ and PT-INR $(\mathrm{p}=0.006)$ than those of antithrombotic naive patients.

No statistically significant difference was observed in the number of patients with any complications between patients undergoing CAT (63\%) and antithrombotic naive patients (73\%). Comparisons of each postoperative complication (bleeding, infection, and subcutaneous emphysema) also revealed no significant differences between the two groups (Table 3).

\subsection{Risk factors for clinical complications after ST}

Table 4 summarizes the characteristics of patients with or without complications. Among the 49 patients (17\%) who developed complications within 7 days after ST, 11 (4\%) had bleeding, 29 (10\%) had infection, and $16(3 \%)$ had subcutaneous emphysema. ST under local anesthesia and ST for airway emergency significantly increased the risk of complications after ST $(\mathrm{p}=0.01$ and $\mathrm{p}=0.02$, respectively). Among the complications, subcutaneous emphysema was significantly more frequent in ST under local anesthesia $(\mathrm{p}<0.001)$ and under airway emergency $(\mathrm{p}=0.049)$ (Table 5). 


\section{DISCUSSION}

In the present study, we investigated the safety of ST in patients undergoing CAT and arrived at the following two major results. First, no significant difference was observed in the incidence of postoperative complications with or without antithrombotic medication, even though APTT and PT-INR showed significant differences among these patients. Second, ST under local anesthesia and airway emergency was a risk factor for complications after ST.

The literatures contain inconsistent reports regarding the additive risk of postoperative bleeding with the use of antithrombotic drugs, and this could presumably be attributed to the differences in surgical procedures, patient demographics, and degree of antithrombotic conditions among patients included in the various studies. For instance, the risk of chronic bleeding after percutaneous dilatational tracheostomy (PDT) is reportedly higher in the presence of two or more abnormal coagulation variables ${ }^{13}$ even though CAT is not considered a risk factor for bleeding after PDT. ${ }^{14}$ CAT also increased the frequency of hemorrhagic complications requiring intervention after prostate biopsy. ${ }^{15}$ In contrast, a study reported no association between the risk of bleeding after percutaneous endoscopic gastrostomy and CAT; pulmonary procedures under CAT did not increase the risk of bleeding either. ${ }^{16}$ Various reports have also concluded that CAT is not a risk factor for bleeding after minimally invasive surgeries, such as tooth extraction, percutaneous endoscopic gastrostomy, and endoscopic variceal ligation. ${ }^{7,11,12}$ The results of the present study support these findings and suggest that ST can be safely performed even under CAT. However, a bias may exist in the differences in the intensity of hemostasis between ST with or without CAT, because surgeons may have performed hemostatic procedures more carefully in ST under CAT to prevent intraoperative and postoperative bleeding.

Based on the present results, we recommend that surgeons should pay careful attention to complications during ST under local anesthesia and in airway emergencies. Awake tracheostomy is 6.2 times more likely to result in pneumothorax or pneumomediastinum than general tracheostomy. ${ }^{5}$ Patients undergoing awake tracheostomy often breathe spontaneously after tracheostomy and are prone to the cough reflex due to the presence of a tracheal cannula, which may result in subcutaneous emphysema. Particularly, in airway emergencies, the risk of subcutaneous emphysema may have been increased because securing the airway is a top priority, despite the consideration of safety. However, no significant intergroup differences were observed in the risk of postoperative infection. Considering that prophylactic administration of antibiotics can prevent the incidence of PDT-induced wound infection, ${ }^{17}$ the use of antibiotics for the treatment of the primary disease may have reduced the incidence of infection after ST. Other potential risk factors, such as DM or albumin level, did not affect the complication rate in the present study. DM has been reported to be a risk factor for surgical site infection (SSI) and for postoperative infection, ${ }^{18}$ and compared to a normal albumin level, a low albumin level $(<3.5 \mathrm{~g} / \mathrm{dL})$ has been associated with an approximately 2.5 times increased risk of SSI in orthopedic surgery. ${ }^{19}$ Nevertheless, these factors may not play an important role in minimally invasive surgeries like ST.

Several limitations of this study should be acknowledged. Retrospective chart reviews are limited by incomplete or missing documentation. Patients undergoing ST performed by surgeons at the other departments were excluded from this analysis, and this might have resulted in the underestimation of the overall incidence of postoperative complications. Moreover, patients undergoing PDT, which involves placing a tracheostoma by dilating the tracheal wall, were excluded from this study. When bleeding occurs during and after PDT, it might be difficult to identify the bleeding site, but in ST, the bleeding site can be easily identified and hemostasis procedures can be performed immediately. Finally, long-term postoperative complications were not investigated in this study.

In the clinical setting, ST can be performed relatively safely regardless of the use of CAT. However, the incidence of complications can be high in cases of ST performed under local anesthesia or airway emergencies. Thus, in such cases, ST should be performed carefully.

\section{CONCLUSION}

The current study suggests that CAT does not increase the risk of postoperative complications after ST and 
that ST can be performed relatively safely regardless of the use of antithrombotic drugs. Nevertheless, when ST is performed under local anesthesia without intubation and for emergency airway access, surgeons should practice extra caution to prevent the occurrence of postoperative complications.

\section{References}

1. Goldenberg D, Ari EG, Golz A, Danino J, Netzer A, Joachims HZ. Tracheotomy complications: a retrospective study of 1130 cases. Otolaryngology-head and neck surgery : official journal of American Academy of Otolaryngology-Head and Neck Surgery 2000; 123:495-500.

2. Durbin CG, Jr. Early complications of tracheostomy. Respiratory care 2005; 50:511-515.

3. Halum SL, Ting JY, Plowman EKet al. A multi-institutional analysis of tracheotomy complications. The Laryngoscope 2012; 122:38-45.

4. Barrera SC, Sanford EJ, Ammerman SB, Ferrell JK, Simpson CB, Dominguez LM. Postoperative Complications in Obese Patients After Tracheostomy. OTO open 2020; 4:2473974X20953090.

5. Bathula SS, Srikantha L, Patrick T, Stern NA. Immediate Postoperative Complications in Adult Tracheostomy. Cureus 2020; 12:e12228.

6. Brenner MJ, Pandian V, Milliren CEet al. Global Tracheostomy Collaborative: data-driven improvements in patient safety through multidisciplinary teamwork, standardisation, education, and patient partnership. British journal of anaesthesia 2020; 125:e104-e118.

7. Perry DJ, Noakes TJ, Helliwell PS, British Dental S. Guidelines for the management of patients on oral anticoagulants requiring dental surgery. British dental journal 2007; 203:389-393.

8. van Diermen DE, Aartman IH, Baart JA, Hoogstraten J, van der Waal I. Dental management of patients using antithrombotic drugs: critical appraisal of existing guidelines. Oral surgery, oral medicine, oral pathology, oral radiology, and endodontics 2009; 107:616-624.

9. Little JW, Miller CS, Henry RG, McIntosh BA. Antithrombotic agents: implications in dentistry. Oral surgery, oral medicine, oral pathology, oral radiology, and endodontics 2002; 93:544-551.

10. Scully C, Wolff A. Oral surgery in patients on anticoagulant therapy. Oral surgery, oral medicine, oral pathology, oral radiology, and endodontics 2002; 94:57-64.

11. Barton CA, McMillian WD, Osler Tet al. Anticoagulation management around percutaneous bedside procedures: is adjustment required? The journal of trauma and acute care surgery 2012; 72:815-820; quiz 1124-1125.

12. Boustiere C, Veitch A, Vanbiervliet Get al. Endoscopy and antiplatelet agents. European Society of Gastrointestinal Endoscopy (ESGE) Guideline. Endoscopy 2011; 43:445-461.

13. Beiderlinden M, Eikermann M, Lehmann N, Adamzik M, Peters J. Risk factors associated with bleeding during and after percutaneous dilational tracheostomy. Anaesthesia 2007; 62:342-346.

14. Pasin L, Frati E, Cabrini Let al. Percutaneous tracheostomy in patients on anticoagulants. Annals of cardiac anaesthesia 2015; 18:329-334.

15. Tanabe K, Hattori T, Kobayashi Het al. Is it safe to continue antithrombotic agents before prostate biopsy? Prostate international 2019; 7:78-81.

16. Herman DD, Thomson CC, Brosnhan Set al. Risk of bleeding in patients undergoing pulmonary procedures on antiplatelet or anticoagulants: A systematic review. Respiratory medicine 2019; 153:76-84.

17. Sittitrai P, Siriwittayakorn C. Perioperative antibiotic prophylaxis in open tracheostomy: A preliminary randomized controlled trial. International journal of surgery 2018; 54:170-175. 
18. Yuwen P, Chen W, Lv Het al. Albumin and surgical site infection risk in orthopaedics: a meta-analysis. BMC surgery 2017; 17:7.

19. Martin ET, Kaye KS, Knott Cet al. Diabetes and Risk of Surgical Site Infection: A Systematic Review and Meta-analysis. Infection control and hospital epidemiology 2016; 37:88-99.

\section{Hosted file}

Table 1.docx available at https://authorea.com/users/426123/articles/530738-safety-ofsurgical-tracheostomy-under-continued-antithrombotic-therapy

\section{Hosted file}

Table 2.docx available at https://authorea.com/users/426123/articles/530738-safety-ofsurgical-tracheostomy-under-continued-antithrombotic-therapy

\section{Hosted file}

Table 3.docx available at https://authorea.com/users/426123/articles/530738-safety-ofsurgical-tracheostomy-under-continued-antithrombotic-therapy

\section{Hosted file}

Table 4.docx available at https://authorea.com/users/426123/articles/530738-safety-ofsurgical-tracheostomy-under-continued-antithrombotic-therapy

\section{Hosted file}

Table 5.docx available at https://authorea.com/users/426123/articles/530738-safety-ofsurgical-tracheostomy-under-continued-antithrombotic-therapy 